УДК 550.831.015: 550.831.23: 519.654

\title{
СРАВНЕНИЕ ГЛОБАЛЬНЫХ МОДЕЛЕЙ ГРАВИТАЦИОННОГО ПОЛЯ НА ТЕРРИТОРИИ МАГАДАНСКОЙ ОБЛАСТИ
}

\author{
Хасанов И. М. ${ }^{1}$, Муравьев Л. А. ${ }^{2}$ \\ ${ }^{I}$ ФББУН Северо-Восточный комплексный научно-исследовательский институт \\ им. Н. А. Шило ДВО РАН, г. Магадан \\ ${ }^{2}$ Институт геофизики УрО РАН, г. Екатеринбург \\ Email: aumaglan@yandex.ru; mlev@mail.ru
}

\begin{abstract}
Общемировые базы данных гравитационного поля, доступные в настоящее время исследователям, представляют новый и информативный инструмент для построения плотностных моделей глубинного строения земной коры в масштабах отдельных регионов. Сейчас существует 6 моделей гравитационных аномалий, представленных в виде разложения по сферическим гармоникам до 2190-й степени, что соответствует около 10 км на поверхности Земли. Разные методы обработки доступных их авторам земных, морских, аэро- и спутниковых гравиметрических данных обусловливают различие между этими моделями как в общеземном масштабе, так и в рамках конкретных регионов. На территорию Магаданской области мы выполнили сравнение моделей EGM2008, GECO, EIGEN-6C4 и WGM2012 с данными картографического материала Гравимаг. Сравнение показало, что общемировые модели гравитационного поля можно использовать в качестве исходных данных для построения плотностных моделей и изучения глубинного строения литосферы в региональном масштабе. Аномалии в свободном воздухе для моделей EGM2008, GECO, EIGEN-6C4 и WGM2012 на выбранной территории практически совпадают. Аномалии Буге модели WGM2012 могут использоваться для целей полнотного моделирования на сопредельных территориях, где отсутствуют кондиционные наземные гравиметрические материалы, однако в рамках территории Магаданской области база Гравимаг обладает наилучшим качеством исходных данных.
\end{abstract}

Ключевые слова: гравитационное поле Земли, региональное гравитационное поле, общемировые базы геоданных, сферические гармоники, аномалия силы тяжести, сравнительный анализ, точность, Арктика.

DOI: $10.34078 / 1814-0998-2021-3-82-89$

\section{ВВЕДЕНИЕ}

Потенциальные поля, наблюдаемые с земной поверхности, раскрывают особенности глубинного строения Земли. Данные гравитационного поля используются при построении моделей литосферы в дополнение к сейсмическим и электромагнитным исследованиям по региональным профилям и позволяют получать информацию о распределении плотностных неоднородностей земной коры в межпрофильном пространстве. Данные космических миссий Lageos, GRACE и GOCE, а также интеграция данных наземных, морских, авиационных гравитационных съемок позволили создать несколько общемировых моделей аномалий гравитационного поля, представленных разложением по сферическим гармони-

(С Хасанов И. М., Муравьев Л. А., 2021 кам. Сейчас созданы модели, имеющие наибольшую степень гармоник 2190, что соответствует примерно 10 км на земной поверхности. Модели различаются набором исходных данных и методов их обработки, и возникает вопрос об их применимости для исследования глубинного строения литосферы в рамках отдельных регионов. Мы проанализировали современные ультравысокостепенные модели гравитационных аномалий на территории Магаданской области, сравнив их с результатами наземной съемки картографического материала Гравимаг.

\section{ИСХОДНЫЕ ДАННЫЕ}

В настоящее время данные глобальных моделей гравитационного поля аккумулируются на сайте отделения Потсдамского центра имени Гельмгольца - GFZ-Potsdam: International Cen- 
tre for Global Earth Models (Международный центр глобальных моделей Земли). Калькулятор данного сайта позволяет получить распределение гравитационных аномалий на любой участок с заданной детальностью (Ince et al., 2019). Сейчас сайт содержит данные о 177 статических моделях поля, шесть из которых имеют степень гармоник 2190. Первая из таких моделей - EGM2008 (Pavlis et al., 2008). Последующие модели были уточнены за счет включения более современных и детальных спутниковых и наземных данных. В настоящей статье мы рассматриваем модели EIGEN-6C4 (Förste et al., 2014), GECO (Gilardoni et al., 2016) и базу данных WGM2012, реализованную Международным бюро гравиметрии (BGI) и принятую Комиссией по геологической карте мира (CGMW) в качестве мировой карты гравитационных аномалий. Данные получены на основе моделей EGM2008 и DTU10 с вычислением полной сферической редукции Буге (Balmino et al., 2012) на основе рельефа земной поверхности ЕТОРО1, представленного сферическими гармониками до степени 10800. Также доступна база данных аномалий в свободном воздухе.

Достоверность моделей EGM2008, EIGEN6C4 и GECO в сравнении между собой и с материалами наземных и морских гравитационных съемок разных районов была проанализирована сотрудниками ИФЗ РАН (Конешов, Непоклонов, 2018; Конешов и др., 2019).

Ранее на территории Магаданской области (Muravyev, Hasanov, 2019) и Уральского региона (Муравьев, 2019) нами была проализирована опубликованная в 2018 г. китайскими учеными модель SGG-UGM-1, имеющая степень сферических гармоник 2159. Для выбранных нами регионов России эта модель оказалась абсолютно некондиционной. Вероятно, вычисления коэффициентов перед сферическими гармониками при формировании данной модели были проведены так, чтобы модель отвечала данным на территории Китая, а данные на другие регионы не использовались или были учтены неверно. В 2020 г. появилась вторая версия данной модели, которую еще предстоит проанализировать, - SGG-UGM-2.

Также ранее на территорию Магаданской области мы проанализировали (Muravyev, Hasanov, 2019) «экспериментальную» модель XGM2019e_2159, которая также не показала улучшения по сравнению с рассмотренными ранее моделями.

Таким образом, для сопоставления с наземными данными гравиметрических карт Гравимаг мы выбрали модели EGM2008, EIGEN-6C4, GECO и базу данных WGM2012.

Банк данных Гравимаг представляет собой цифровую модель гравитационного поля, состав- ленную по материалам гравиметрических съемок масштаба 1:200 000. Исходными данными по каждому пункту гравиметрических наблюдений являются значения аномалии силы тяжести в редукции Буге с плотностью промежуточного слоя 2.67 г $/ \mathrm{cm}^{3}$ с введенными поправками за влияние рельефа с радиусом 50-200 км.

Для сопоставления мы выполнили вычисления гравитационных аномалий согласно перечисленным моделям на территорию, локализо-

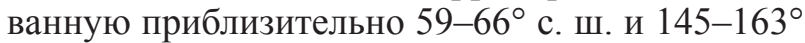
в. д., ограничив полученные сеточные файлы маской по административным границам Магаданской области и частью территории морского шельфа. Все данные были построены в виде сеток $5 \times$ 5 км в условном уровне и координатах.

Калькулятор GFZ-Potsdam позволяет получать гравитационные аномалии в нескольких вариантах (Barthelmes, 2009): классическом, современном и редукции Буге. Разность между гравитационным полем, аналитически продолженным из точки измерения вниз на высоту геоида, и нормальным полем на эллипсоиде дает классическое определение гравитационной аномалии. Поверхность геоида определяется путем решения проблемы Стокса (Молоденский, 1945), поэтому практически для получения величины поля на высоте геоида выполняется редукция за свободный воздух (Barthelmes, 2009).

Современное определение гравитационной аномалии основано на теории Молоденского (Молоденский, 1945) - разность между гравитационным полем в точке и нормальным полем в точке с такими же эллиптическими координатами, но на высоте над эллипсоидом, равной $(\mathrm{h}-\zeta \mathrm{g})$, где $\zeta \mathrm{g}$ - аномалия высоты. Эта величина - расстояние от поверхности Земли до точки, где нормальный потенциал имеет то же значение, что потенциал на поверхности. В этом случае не используется аналитическое продолжение вниз.

Аномалия Буге рассчитывается вычитанием из классической гравитационной аномалии величины $2 \pi \mathrm{G} \rho \mathrm{H}$. Высота Н при этом рассчитывается из модели рельефа ЕTOPO1 (Amante, Eakins, 2009) (рис. 1,d), которая также построена на разложении по сферическим гармоникам. Плотность определяется для положительных высот $\rho=2.670$ г $/ \mathrm{cm}^{3}$, а для отрицательных (акватории) $\rho=(2.670-1.025) \Gamma / \mathrm{cm}^{3}$.

\section{СОПОСТАВЛЕНИЕ МОДЕЛЕЙ}

Карта распределения аномалий в свободном воздухе (классическое определение в терминологии GFZ-Potsdam) согласно четырем моделям - EGM2008, EIGEN-6C4, GECO и WGM2012 приведена на рис. 1,a. Морфология аномалий в целом одинакова, немного отличается средний 

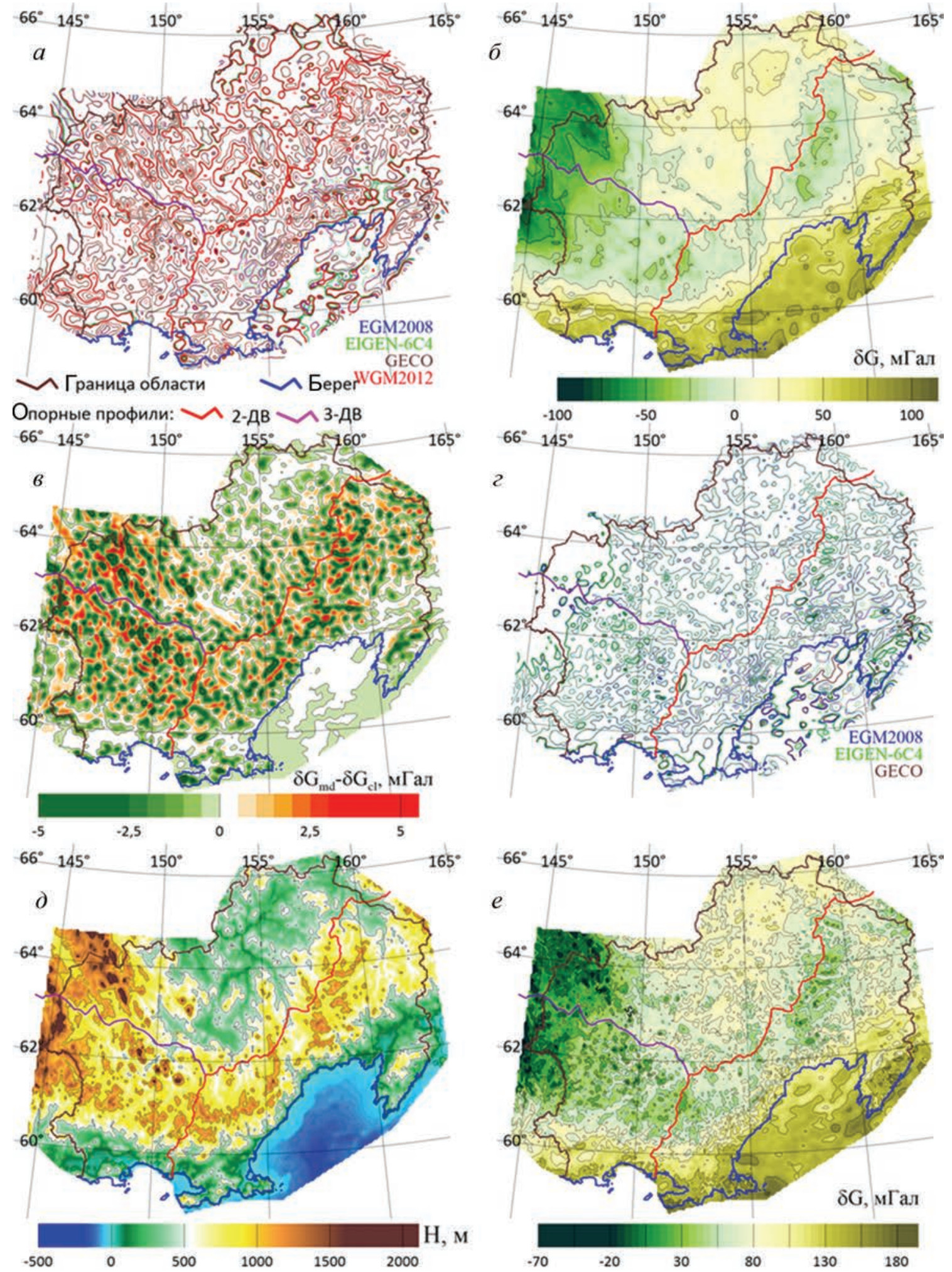

Pис. 1. Гравитационное поле на территорию Магаданской области: $a$ - согласно современным общеземным моделям EGM2008, EIGEN-6C4, GECO, WGM2012 в свободном воздухе; $\sigma$ - картографический материал Гравимаг (Буге); в - разность аномалий в свободном воздухе и согласно современному (по Молоденскому) определению для модели EGM2008; 2 - аномалии Буге моделей EGM2008, EIGEN-6C4, GECO; $\partial$ - рельеф согласно модели ЕТОРО1; e- аномалии Буге WGM2012

Fig. 1. Gravity field on the Magadan Oblast territory: $a$ - free air anomaly according to the EGM2008, EIGEN-6C4, GECO, WGM2012 models; $\sigma$ - Bouguer anomalies according to the Gravimag database; 8 - the difference between anomalies in free air and according to the modern (Molodensky theory) definition for the EGM2008 model; 2 - Bouguer anomalies for the EGM2008, EIGEN-6C4, GECO models; $\partial$ - relief according to the ETOPO1 model; $e-$ WGM2012 Bouguer anomalies 
уровень и имеются небольшие отличия в отдельных частях участка.

Выполненное нами ранее для Уральского региона (Муравьев, 2019) сравнение аномалий моделей EGM2008, EIGEN-6C4 и GECO по классическому и современному (теория Молоденского) определениям показало равенство этих определений. Для горных районов разность между ними не превышала 1 мГал, для основной территории - 0.1 мГал. Для Магаданской области карта разности аномалий в свободном воздухе и согласно современному определению для модели EGM2008 приведена на рис. 1,в. В горных районах разность достигает 5-10 мГал, а на акватории - 0.5 мГал. Для EIGEN-6C4 и GECO результаты сравнения те же.

Изолинии аномалий моделей EGM2008, GECO, EIGEN-6C4 в редукции Буге приведены на рис. 1, г. Аномалии Буге для этих моделей почти совпадают. При этом данные модели
WGM2012 (рис. 1,e) содержат более мелкие аномалии и субъективно представляются более детальными, скорее всего, по причине того, что для редукции были использованы данные рельефа ЕТОРО1 по более детальной сети: $1 \times 1$ угловая минута. Кроме того, аномалии Буге WGM2012 имеют другой средний уровень. Картографические данные Гравимаг также имеют более высокое среднее значение. Статистические данные по моделям приведены на рис. 2, $a$.

Для дальнейшего сравнения моделей между собой мы вычислили сетки средних значений из данных каждой модели отдельно для каждой редукции: свободный воздух, Буге и по современному определению (по Молоденскому).

Для аномалий в свободном воздухе были усреднены четыре сетки - EGM2008, GECO, EIGEN-6C4, WGM2012 и получены разности каждой из сеток с усредненной сеткой.

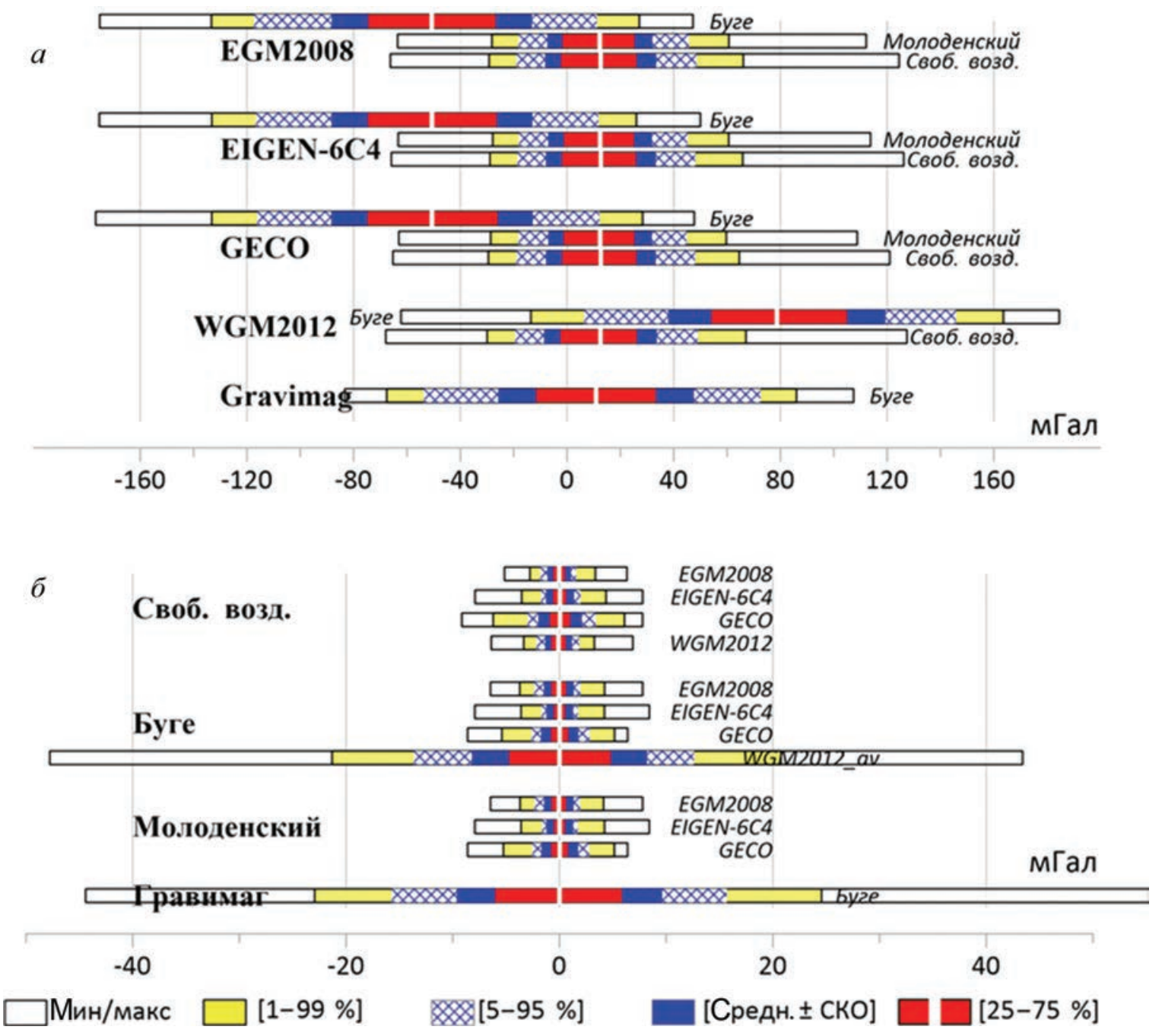

Puc. 2. Статистические характеристики баз данных гравитационных аномалий в пределах территории Магаданской области $(a)$ и разностей между базами данных и сетками усредненных значений для каждой редукции (б)

Fig. 2. Statistical characteristics of gravitational anomaly databases within Magadan Oblast (a) and of the differences between the databases and grids of averaged values for each reduction (б) 
Для аномалий согласно современному определению (по Молоденскому) усреднены EGM2008, GECO, EIGEN-6C4 и аналогично получены разности каждой с усредненной.

Средние по сеткам значения аномалий Буге для разных моделей существенно различаются между собой, поэтому усреднены аномалии Буге трех сеток: EGM2008, GECO, EIGEN-6C4 и при построении разностей с сетками WGM2012 и Гравимаг из нее вычтены также средние по этим сеткам. Статистические характеристики разностей между каждой из моделей и сетками усредненных значений приведены на рис. 2,б.
На рис. 3 показаны графики гравитационных аномалий вдоль опорных геолого-геофизических профилей 2-ДВ и 3-ДВ с поправками за свободный воздух и Буге. На некоторых фрагментах профилей данные всех моделей практически идеально совпадают, но в других частях различия достигают 5 мГал для аномалий в свободном воздухе. На графиках аномалий Буге отчетливо видно, что данные Гравимаг более сглажены, чем WGM2012. Статистические характеристики моделей вдоль данных профилей приведены в таблице.

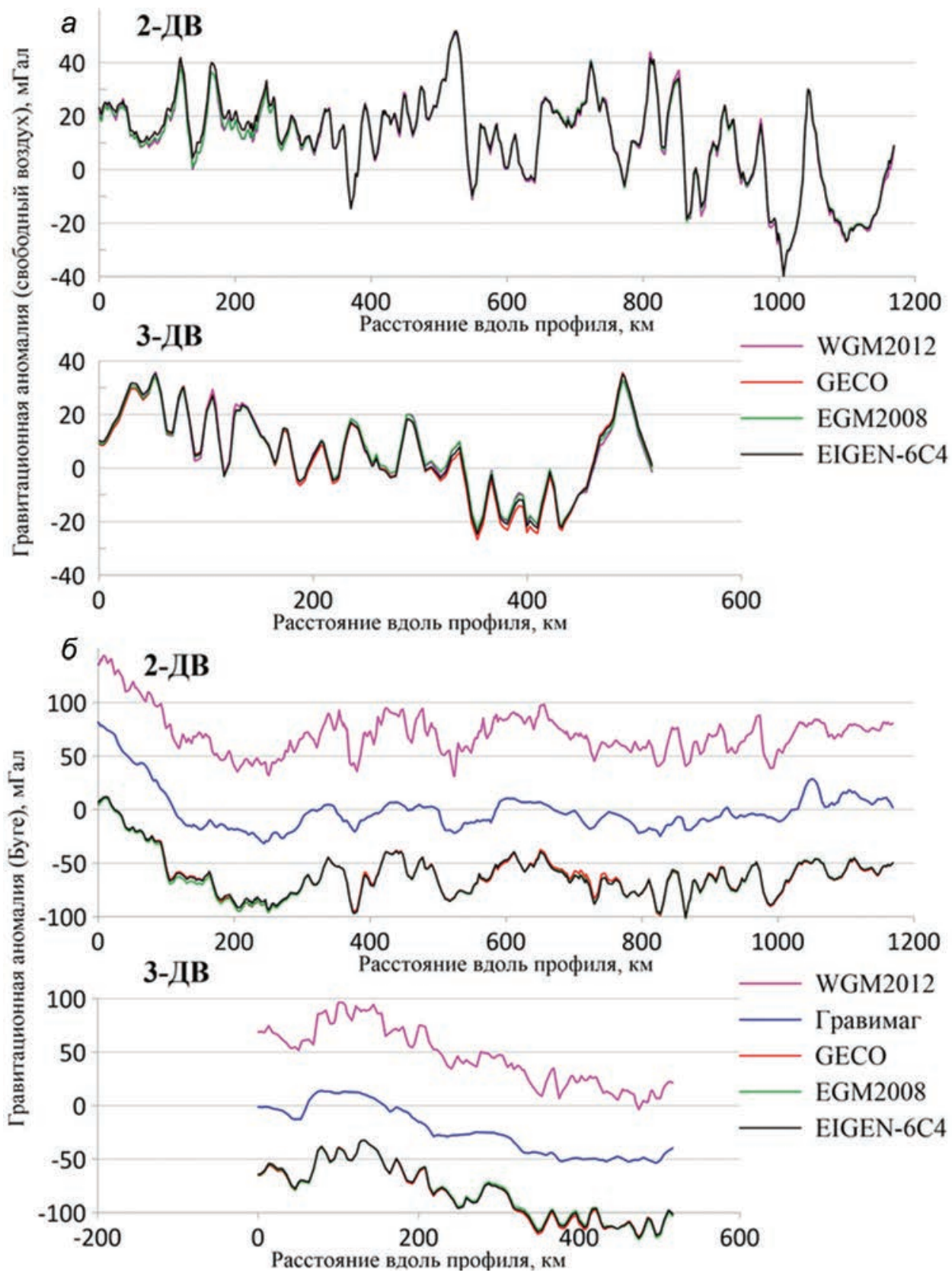

Puc. 3. Сравнение гравитационных аномалий по глобальным моделям вдоль профилей глубинного зондирования 2-ДВ и 3-ДВ в свободном воздухе (a) и Буге (б)

Fig. 3. Comparison of gravity anomalies by global models along the 2-DV and 3-DV deep geophysical sounding profiles in reductions: free air $(a)$ and Bouguer $(\sigma)$ 
Таблища. Статистические характеристики баз данных гравитационных аномалий вдоль опорных геологогеофизических профилей 2-ДВ и 3-ДВ

Table. Statistical characteristics of gravity anomaly databases along the 2-DV and 3-DV reference geologicalgeophysical profiles

\begin{tabular}{|c|c|c|c|c|c|c|c|c|}
\hline БД & $\begin{array}{c}\text { Ре- } \\
\text { дук- } \\
\text { ция }\end{array}$ & Мин & Макс & Среднее & Ст. откл. & Медиана & 1-й квартиль & 3-й квартиль \\
\hline \multicolumn{9}{|c|}{ Профиль 2-ДВ } \\
\hline EGM2008 & \multirow{4}{*}{ 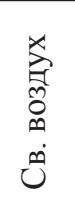 } & -39.7 & 51.5 & 10.3 & 12.8 & 0.9 & 21.3 & 16.4 \\
\hline EIGEN-6C4 & & -39.8 & 51.8 & 11.1 & 14.2 & 1.5 & 22 & 16.8 \\
\hline GECO & & -40.9 & 52.3 & 11 & 13.6 & 1.7 & 22.6 & 17.2 \\
\hline WGM2012 & & 31 & 143.2 & 71.3 & 69.6 & 57.1 & 81 & 20.9 \\
\hline EGM2008 & \multirow{5}{*}{ 疋 } & -101.7 & 10.3 & -61.7 & -63.8 & -76.6 & -51.7 & 21 \\
\hline EIGEN-6C4 & & -100.7 & 12.1 & -60.9 & -63.2 & -75.9 & -51.5 & 20.8 \\
\hline GECO_BG & & -101.5 & 10.6 & -61 & -62.3 & -76.4 & -51.6 & 21 \\
\hline WGM2012 & & 31 & 143.2 & 71.3 & 69.6 & 57.1 & 81 & 20.9 \\
\hline Гравимаг & & -31.6 & 81.7 & -0.4 & -4.6 & -14.2 & 6.6 & 20.5 \\
\hline \multicolumn{9}{|c|}{ Профиль 3-ДВ } \\
\hline EGM2008 & \multirow{4}{*}{ 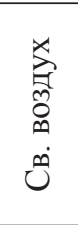 } & -23 & 34.1 & 5.9 & 14.1 & 6.9 & -2.8 & 16.2 \\
\hline EIGEN-6C4 & & -24.7 & 35.4 & 5.7 & 14.9 & 5.3 & -3.7 & 16.1 \\
\hline GECO & & -26.8 & 35.6 & 4.9 & 15.3 & 5.3 & -4.9 & 16 \\
\hline WGM2012 & & -24.1 & 35.8 & 5.8 & 14.4 & 5.9 & -2.9 & 16.2 \\
\hline EGM2008 & \multirow{5}{*}{ 蛋 } & -125.2 & -32.7 & -81.8 & 25.2 & -79.4 & -106.6 & -60.5 \\
\hline EIGEN-6C4 & & -123.6 & -32.4 & -82.1 & 25.5 & -80.4 & -106.4 & -59.8 \\
\hline GECO_BG & & -122.8 & -32.4 & -82.8 & 25.7 & -81.2 & -106.4 & -61.1 \\
\hline WGM2012 & & -3.6 & 96.5 & 45.4 & 27 & 45.3 & 21.8 & 68.1 \\
\hline Гравимаг & & -53.6 & 13.8 & -24.3 & 22.7 & -27.4 & -47.5 & -3.6 \\
\hline
\end{tabular}

\section{ЗАКЛЮЧЕНИЕ}

Сравнение общеземных моделей гравитационных аномалий для территории Магаданской области показало, что из доступных на настоящий момент моделей именно модели EGM2008, GECO, EIGEN-6C4 и WGM2012 кондиционны и могут служить исходными данными для построения плотностных моделей глубинного строения литосферы в региональном масштабе. Аномалии в свободном воздухе для моделей EGM2008, GECO, EIGEN-6C4 и WGM2012 на выбранной территории практически совпадают. Аномалии Буге модели WGM2012 могут использоваться для целей полнотного моделирования на сопредельных территориях, где отсутствуют кондиционные наземные гравиметрические материалы. Это особенно актуально для исследования региона приполярного шельфа Северо-Востока России как территорий, перспективных на поиски месторождений твердых полезных ископаемых и углеводородного сырья.

\section{ЛИТЕРАТУРА}

Конешов В. Н., Непоклонов В. Б. Исследование точности представления гравитационного поля Земли в полярных районах по данным глобальных моделей геопотенциала // Физика Земли. 2018. № 3. С. 123 131. DOI:10.7868/S0002333718030092

Конешов В. Н., Непоклонов В. Б., Соловьев В. Н., Железняк Л. К. Сравнение современных глобальных ультравысокостепенных моделей гравитационного поля Земли // Геофизические исследования. 2019. № 1. C. 13-26. DOI:10.21455/gr2019.1-2

Молоденский M. С. Основные вопросы геодезической гравиметрии. Москва : Геодезиздат, 1945. 111 с. (Труды ЦНИИГАиК ; вып. 42).

Муравьев Л. А. Общеземные базы данных гравитационного поля Земли на территорию приарктической части Уральского региона // Уральский геофизический вестник. 2019. № 2 (36). С. 46-53. DOI:10.25698/ UGV.2019.2.5.46

Amante C., Eakins B. W. ETOPO1 1 arc-minute global relief model: Procedures, data sources and analysis. NOAA Technical Memorandum NESDIS NGDC-24. National Geophysical Data Center, NOAA, 2009. DOI: 10.7289/V5C8276M 
Balmino G., Vales N., Bonvalot S., Briais A. Spherical harmonic modeling to ultra-high degree of Bouguer and isostatic anomalies // Journal of Geodesy. 2012. Vol. 86 (7). P. 499-520. DOI: 10.1007/s00190-011-0533-4

Barthelmes $F$. Definition of functionals of the geopotential and their calculation from spherical harmonic models: theory and formulas used by the calculation service of the International Centre for Global Earth Models (ICGEM). Scientific Technical Report 09/02. German Research Centre for Geosciences (GFZ), 2009. DOI: 10.2312/GFZ.b103-0902-26

Förste C., Bruinsma S. L., Abrikosov O., Lemoine J.-M., Marty J. C., Flechtner F., Balmino G., Barthelmes $F$., Biancale R. EIGEN-6C4 The latest combined global gravity field model including GOCE data up to degree and order 2190 of GFZ Potsdam and GRGS Toulouse. GFZ Data Services, 2014. DOI: 10.5880/ICGEM.2015.1

Gilardoni M., Reguzzoni M., Sampietro D. GECO: A global gravity model by locally combining GOCE data and EGM2008 // Studia Geophysica et Geodaetica. 2016. Vol. 60. P. 228-247. DOI: 10.1007/s11200-015-1114-4
Hasanov I. M., Muravyev L. A. Comparison of the new gravity field model XGM2019e with other modern global models of the gravitational field for the Magadan Region / $19^{\text {th }}$ International Conference on Geoinformatics - Theoretical and Applied Aspects. 2020. DOI 10.3997/2214-4609.2020geo112

Ince E. S., Barthelmes F., Reißland S., Elger K., Förste $C$., Flechtner F., Schuh H. ICGEM - 15 years of successful collection and distribution of global gravitational models, associated services, and future plans // Earth System Science Data. 2019. Vol. 11. P. 647-674. DOI:10.5194/ essd-11-647-2019

Muravyev L. A., Hasanov I. M. Comparison of global gravity field databases for the territory of the Magadan Region / $18^{\text {th }}$ International Conference on Geoinformatics - Theoretical and Applied Aspects. 2019. DOI:10.3997/2214-4609.201902135

Pavlis N. K., Holmes S. A., Kenyon S. C., Factor $J$. $K$. An earth gravitational model to degree 2160: EGM2008 / EGU General Assembly 2008.Vienna, Austria. April 13-18, 2008.

\title{
COMPARISON OF GLOBAL MODELS OF THE GRAVITATIONAL FIELD IN THE TERRITORY OF THE MAGADAN OBLAST
}

\author{
I. M. Khasanov', L. A. Muravyev ${ }^{2}$ \\ ${ }^{1}$ North-East Interdisciplinary Scientific Research Institute n. a. N. A. Shilo, FEB RAS, Magadan \\ ${ }^{2}$ Institute of Geophysics UB RAS, Yekaterinburg
}

\begin{abstract}
The global databases of gravity anomalies, currently available to researchers, provide a new informative tool for constructing density models of the deep structure of the earth's crust for individual regions. Currently, there are six models of gravitational anomalies, presented as a series of spherical harmonics up to 2190 degrees, which corresponds to about $10 \mathrm{~km}$ on the earth's surface. Different methods of processing terrestrial, marine, aerial, and satellite gravimetric data, available to their authors, determine the differences between these models, both on a global scale and within specific regions. We have performed a comparison of the EGM2008, GECO, EIGEN-6C4, and WGM2012 models with the Gravimag database on the Magadan Oblast territory. The comparison showed that free air anomalies for the EGM2008, GECO, EIGEN-6C4, and WGM2012 models in the selected area almost coincide. Bouguer anomalies of the WGM2012 model can be used in regional density modeling for adjacent regions where there are no conventional ground gravity data; however, within Magadan Oblast the Gravimag database has the best data quality.
\end{abstract}

Keywords: earth's gravitational field, regional gravitational field, global databases, spherical harmonics, gravity anomaly, comparative analysis, accuracy, Arctic.

\section{REFERENCES}

Amante, C., Eakins, B. W., 2009. ETOPO1 1 ArcMinute Global Relief Model: Procedures, Data Sources and Analysis. NOAA Technical Memorandum NESDIS NGDC-24. National Geophysical Data Center, NOAA. DOI: $10.7289 / \mathrm{V} 5 \mathrm{C} 8276 \mathrm{M}$

Balmino, G., Vales, N., Bonvalot, S., Briais, A., 2012. Spherical Harmonic Modeling to Ultra-High Degree of Bouguer and Isostatic Anomalies, Journal of Geodesy. 86, 7, 499-520. DOI: 10.1007/s00190-011-0533-4
Barthelmes, F., 2009. Definition of Functionals of the Geopotential and Their Calculation from Spherical Harmonic Models: Theory and Formulas Used by the Calculation Service of the International Centre for Global Earth Models (ICGEM). Scientific Technical Report 09/02, German Research Centre for Geosciences (GFZ). DOI: 10.2312/GFZ.b103-0902-26

Förste, C., Bruinsma, S. L., Abrikosov, O., Lemoine, J.-M., Marty, J. C., Flechtner, F., Balmino, G., Barthelmes, 
F., Biancale, R., 2014. EIGEN-6C4 The Latest Combined Global Gravity Field Model Including GOCE Data up to Degree and Order 2190 of GFZ Potsdam and GRGS Toulouse. GFZ Data Services. DOI: 10.5880/ ICGEM.2015.1

Gilardoni, M., Reguzzoni, M., Sampietro, D., 2016. GECO: a Global Gravity Model by Locally Combining GOCE Data and EGM2008, Studia Geophysica et Geodaetica. 60, 228-247. DOI: 10.1007/s11200-0151114-4

Hasanov, I. M., Muravyev, L. A., 2020. Comparison of the New Gravity Field Model XGM2019e with Other Modern Global Models of the Gravitational Field for the Magadan Region, $19^{\text {th }}$ International Conference on Geoinformatics - Theoretical and Applied Aspects. DOI: 10.3997/2214-4609.2020geo112

Ince, E. S., Barthelmes, F., Reißland, S., Elger, K., Förste, C., Flechtner, F., Schuh, H., 2019. ICGEM - 15 Years of Successful Collection and Distribution of Global Gravitational Models, Associated Services, and Future Plans, Earth System Science Data. 11, 647-674. DOI: 10.5194/essd-11-647-2019

Koneshov, B. N., Nepoklonov, V. B., 2018. Research of the Representation Accuracy of the Earth's Gravity
Field in the Polar Areas by the Data from the Global Geopotential Models, Izvestiya. Physics of the SolidEarth. 54, 3, 504-512. DOI:10.1134/S1069351318030047 [In Russian].

Koneshov, B. N., Nepoklonov, V. B., Solovyov, V. N., Jeleznyak, L. K., 2019. Comparison of Modern Global Ultra-High-Degree Models of the Earth Gravitational Field, Geophysical Research. 20, 1, 13-26. DOI:10.21455/ gr2019.1-2 [In Russian].

Molodensky, M. S., 1945. Basic Issues in Geodetic Gravimetry. Moscow, Geodezizdat [In Russian].

Muravyev, L. A., 2019. Global Databases of the Earth's Gravitational Field for the Arctic Part of the Urals, Uralskiy Geofizicheskiy Vestnik. 2, 46-53. DOI: 10.25698/ UGV.2019.2.5.46 [In Russian].

Muravyev, L. A., Hasanov, I. M., 2019. Comparison of Global Gravity Field Databases for the Territory of the Magadan Region, $18^{\text {th }}$ International Conference on Geoinformatics - Theoretical and Applied Aspects. DOI: 10.3997/2214-4609.201902135

Pavlis, N. K., Holmes, S. A., Kenyon, S. C., Factor, J. K., 2008. An Earth Gravitational Model to Degree 2160: EGM2008, EGU General Assembly 2008. Vienna, Austria, April 13-18. 